\title{
Stabilization of a Coupled Multidimensional System
}

\author{
Serge NiCAISE and Abdoulaye SÈnE
}

Université de Valenciennes et du Hainaut Cambrésis, MACS, Institut des Sciences et Techniques

F-59313 Valenciennes Cedex 9 - France

snicaise@univ-valenciennes.fr

\author{
Université Cheikh Anta DIOP \\ Département de Mathématiques \\ Faculté des Sciences et Techniques \\ Dakar — Sénégal \\ abdousen@ucad.sn
}

Received: July 7, 2005

Accepted: January 10, 2006

\begin{abstract}
We introduce a model of a vibrating multidimensional structure made of a $n$ dimensional body and a one-dimensional rod. We actually consider the anisotropic elastodynamic system in the $n$-dimensional body and the Euler-Bernouilli beam in the one-dimensional rod. These equations are coupled via their boundaries. Using appropriate feedbacks on a part of the boundary we show the exponential decay of the energy of the system.
\end{abstract}

Key words: multidimensional structures, stabilization, exponential decay.

2000 Mathematics Subject Classification: 93D15, 93B05, 93C20.

\section{Introduction}

Let $\Omega$ be a non empty bounded open subset of $\mathbb{R}^{n}, n \geq 1$, with a boundary $\Gamma$ of class $C^{2}$. We denote by $\nu(x)=\left(\nu_{1}, \ldots, \nu_{n}\right)^{\top}$ the unit outward normal vector at $x$ along $\Gamma$. For a fixed $x_{0} \in \mathbb{R}^{n}$ we define the function $m(x)=x-x_{0}, x \in \mathbb{R}^{n}$ and the following partition of the boundary $\Gamma$ (see figures 1 and 2 ):

$$
\begin{aligned}
\Gamma_{0} & =\{x \in \Gamma: m(x) \cdot \nu(x) \leq 0\}, \\
\Gamma_{N} & =\{x \in \Gamma: m(x) \cdot \nu(x)>0\} .
\end{aligned}
$$




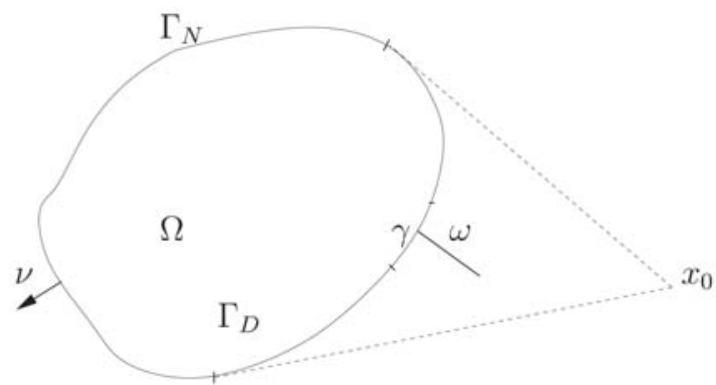

Figure 1: A pluridimensional structure for $n=2-$ The case $\bar{\Gamma}_{N} \cap \bar{\Gamma}_{D} \neq \emptyset$

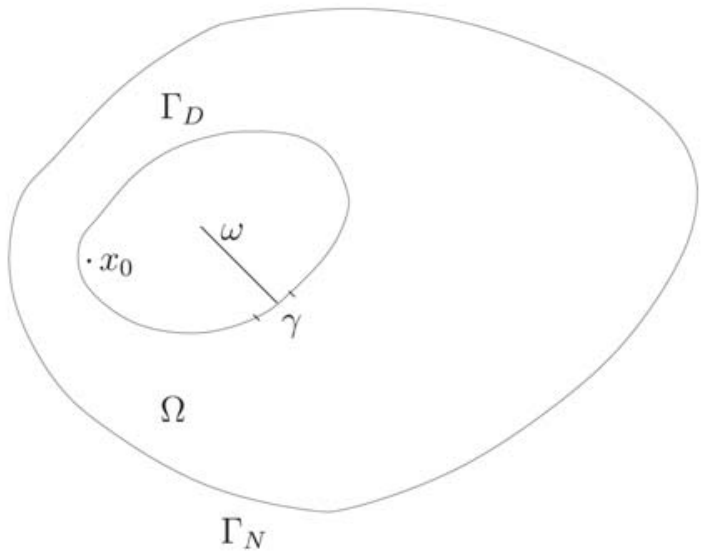

Figure 2: A pluridimensional structure for $n=2-$ The case $\bar{\Gamma}_{N} \cap \bar{\Gamma}_{D}=\emptyset$ 
We also fix an open subset $\gamma$ of $\Gamma_{0}$ such that

$$
m(x) \cdot \nu(x) \leq-\alpha_{0}<0, \quad \forall x \in \gamma
$$

and denote

$$
\Gamma_{D}=\Gamma_{0} \backslash \gamma
$$

In the whole paper we suppose that meas $\Gamma_{D}>0$, meas $\Gamma_{N}>0$, meas $\gamma>0$.

We further fix a 1-dimensional beam $\omega$ of length $l$ attached to $\Omega$ at a point $a \in \gamma$ and orthogonal to $\Gamma$, in other words (see again figures 1 and 2 ),

$$
\omega=\{a+s \nu(a): 0<s<l\} .
$$

The derivation with respect to the parameter $s$ will be denoted by $\partial$.

Finally let $\alpha$ be a non negative real number and $\theta$ be a function from $\gamma$ to $\mathbb{R}^{n}$ of class $C^{1}$ with a compact support and such that $\theta \neq 0$.

We now consider the following problem:

$$
\begin{cases}u^{\prime \prime}-\operatorname{div} \sigma(u)=0 & \text { in } \Omega \times \mathbb{R}^{+} \\ v^{\prime \prime}+\rho \partial^{4} v=0 & \text { in } \omega \times \mathbb{R}^{+} \\ u=0 & \text { on } \Gamma_{D} \times \mathbb{R}^{+} \\ \sigma(u) \cdot \nu+m \cdot \nu u^{\prime}=0 & \text { on } \Gamma_{N} \times \mathbb{R}^{+} \\ u(x, t)=v(0, t) \theta(x) & \text { on } \gamma \times \mathbb{R}^{+} \\ \rho \partial^{3} v(0, t)+\alpha v^{\prime}(0, t)+\int_{\gamma}[\sigma(u) \cdot \nu] \cdot \theta(x) d s(x)=0 & \forall t \in \mathbb{R}^{+} \\ \partial v(0, t)=\partial^{2} v(l, t)=\partial^{3} v(l, t)=0, & \end{cases}
$$

with initial conditions

$$
\begin{cases}u(0)=u^{0} & \text { in } \Omega, \\ u^{\prime}(0)=u^{1} & \text { in } \Omega, \\ v(0)=v^{0} & \text { in } \omega \\ v^{\prime}(0)=v^{1} & \text { in } \omega,\end{cases}
$$

where, as usual, $u^{\prime}$ means $\frac{\partial u}{\partial t}, u=u(x, t)=\left(u_{1}, \ldots, u_{n}\right)^{\top}$ denotes the displacement vector field in the domain $\Omega$ and $v=v(s, t)$ denotes the orthogonal displacement of the beam $\omega$. The stress tensor $\sigma$ is defined by $\sigma_{i j}(u)=a_{i j k l} \varepsilon_{k l}(u$ ) (in the full paper we adopt the convention of repeated indices), where $\varepsilon(u)$ is the strain tensor given by (when $\left.\partial_{i}=\frac{\partial}{\partial x_{i}}\right)$

$$
\varepsilon_{i j}(u)=\frac{1}{2}\left(\partial_{j} u_{i}+\partial_{i} u_{j}\right)
$$

the constant coefficients $a_{i j k l}$ are such that

$$
a_{i j k l}=a_{k l i j}=a_{j i k l}
$$


and satisfy the ellipticity condition

$$
\exists \delta>0: a_{i j k l} \varepsilon_{i j} \varepsilon_{k l} \geq \delta \varepsilon_{i j} \varepsilon_{i j},
$$

for all symmetric tensor $\varepsilon_{i j}$. Finally $\rho>0$ corresponds to some mechanical properties of the beam $\omega$.

The components of the vector field $\operatorname{div} \sigma(u)$ are given by

$$
(\operatorname{div} \sigma(u))_{i}=\partial_{j} \sigma_{i j}, i=1, \ldots, n .
$$

The system (1) is dissipative since its energy defined by

$$
E(t)=\frac{1}{2} \int_{\Omega}\left\{\left|u^{\prime}\right|^{2}+\sigma(u): \varepsilon(u)\right\} d x+\frac{1}{2} \int_{\omega}\left\{\left|v^{\prime}\right|^{2}+\rho\left|\partial^{2} v\right|^{2}\right\} d s
$$

is non increasing.

If $\bar{\Gamma}_{N} \cap \bar{\Gamma}_{D} \neq \emptyset$, we suppose that the elastodynamical system in $\Omega$ is reduced to the isotropic one, namely we assume that

$$
\sigma(u)=2 \mu \varepsilon(u)+\lambda \operatorname{div} u I_{n},
$$

where $\lambda, \mu>0$ are the Lamé coefficients and $I_{n}$ is the identity matrix in $\mathbb{R}^{n}$. We further need to assume that (cf. [3]) $c:=\bar{\Gamma}_{N} \cap \bar{\Gamma}_{D}$ is a $(n-2)$-dimensional submanifold of class $C^{3}$ such that there exists a neighborhood $\Omega^{\prime}$ of $c$ such that $\Gamma \cap \Omega^{\prime}$ is a $(n-1)$ dimensional submanifold of class $C^{3}$. If $\tau(x)$ denotes the unit normal vector along $c$ pointing outward of $\Gamma_{N}$, we assume that (see figure 1)

$$
m(x) \cdot \tau(x) \leq 0, \quad \forall x \in c .
$$

Note that the above system (1) is a coupled system between the anisotropic elastodynamical system in $\Omega$ and an Euler-Bernouilli beam equation in $\omega$. The feedbacks correspond to the term $m \cdot \nu u^{\prime}$ on $\Gamma_{N}$ and the term $\alpha v^{\prime}(0, t)$ on the junction $\gamma$. (Remark that $\alpha$ may be equal to zero.)

Simpler models were considered in $[19,30,31]$, namely their system is a coupling between the wave equations in $\Omega$ and in $\omega$. In [30,31], the controllability of this system is considered using appropriate control on the boundary; while in [19] the stability of this system is considered with the help of a feedback only on $\Gamma_{N}$. As underlined in [31], the analysis of more realistic models should be made. Therefore our goal is to consider a simple but realistic model of the junction between the elasticity system and a beam. The junction between $\Omega$ and $\omega$ is made via the transmission conditions

$$
\begin{array}{ll}
u(x, t)=v(0, t) \theta(x) & \text { on } \gamma \times \mathbb{R}^{+}, \\
\rho \partial^{3} v(0, t)+\alpha v^{\prime}(0, t)+\int_{\gamma}[\sigma(u) \cdot \nu] \cdot \theta(x) d s(x)=0 & \forall t \in \mathbb{R}^{+} .
\end{array}
$$


The first condition means that the displacement $u$ on $\gamma$ and $v$ at its extremity $a$ is prescribed via the profile $\theta$, in a certain sense the beam is clamped at the domain $\Omega$ since we add the condition $\partial v(0)=0$. The second condition is a (energy) balance law. The boundary conditions on the other extremity of the beam mean that the beam is free at that point. Note that the junction between $\Omega$ and $\omega$ is made through the profile $\theta$, therefore the angle between $\omega$ and the boundary $\Gamma$ of $\Omega$ could be different from $\pi / 2$.

\section{The main results}

We define the following Hilbert spaces:

$$
\begin{aligned}
\mathcal{H} & =\left(L^{2}(\Omega)\right)^{n} \times L^{2}(\omega), \\
H_{\Gamma_{D}}^{1}(\Omega) & =\left\{u \in H^{1}(\Omega): u=0 \text { on } \Gamma_{D}\right\}, \\
V & =\left\{(u, v) \in\left(H_{\Gamma_{D}}^{1}(\Omega)\right)^{n} \times H^{2}(\omega): u=\theta v(0) \text { on } \gamma \text { and } \partial v(0)=0\right\} .
\end{aligned}
$$

The space $V$ is equipped with the natural norm

$$
\|(u, v)\|_{V}^{2}=\int_{\Omega} \sigma(u): \varepsilon(u) d x+\int_{\omega} \rho\left(\partial^{2} v\right)^{2} d s
$$

where $\sigma(u): \varepsilon(u)=\sigma_{i j}(u) \varepsilon_{i j}(u)$.

Theorem 1.1. For the initial data $\left(\left(u_{0}, v_{0}\right),\left(u_{1}, v_{1}\right)\right) \in V \times \mathcal{H}$, the system (1) has a unique (weak) solution $(u, v)$ satisfying

$$
(u, v) \in C^{1}([0, \infty) ; \mathcal{H}) \cap C([0, \infty) ; V) .
$$

The main result of our paper is the next theorem:

Theorem 1.2. There exist positive constants $M$ and $\delta$ such that the energy of any solution of (1) satisfies

$$
E(t) \leq M e^{-\delta t}, \quad \forall t \geq 0 .
$$

Remark 1.3. In [19] the stability of the wave system is obtained under a geometric assumption between $\gamma$ and the length of $\omega$. Our paper shows that this assumption is unnecessary.

\section{Well-posedness of the problem}

In this section we prove Theorem 1.1 by reducing the system (1) to a first order evolution equation.

Let us define the operators

$$
A: V \longmapsto V^{\prime} \quad \text { and } \quad B: V \longmapsto V^{\prime}
$$


by

$$
\begin{aligned}
& \left\langle A(u, v),\left(u^{*}, v^{*}\right)\right\rangle_{V^{\prime}, V}=\int_{\Omega} \sigma(u): \varepsilon\left(u^{*}\right) d x+\int_{\omega} \rho \partial^{2} v \partial^{2} v^{*} d s, \\
& \left\langle B(u, v),\left(u^{*}, v^{*}\right)\right\rangle_{V^{\prime}, V}=\int_{\Gamma_{N}} m \cdot \nu u \cdot u^{*} d \Gamma+\alpha v(0) v^{*}(0) .
\end{aligned}
$$

Clearly the operators $A$ and $B$ are well defined. Now to obtain the abstract formulation of (1), we take an arbitrary element $\left(u^{*}, v^{*}\right) \in V$. We multiply the first identity of the system (1) by $u^{*}$, integrate by parts in $\Omega$, and use the boundary conditions on $\Gamma_{D}$ and $\Gamma_{N}$. This yields

$$
\begin{aligned}
0 & =\int_{\Omega}\left[u^{\prime \prime}-\operatorname{div}(\sigma(u))\right] \cdot u^{*} d x \\
& =\int_{\Omega} u^{\prime \prime} \cdot u^{*} d x-\int_{\Gamma}(\sigma(u) \cdot \nu) \cdot u^{*} d \Gamma+\int_{\Omega} \sigma(u): \varepsilon\left(u^{*}\right) d x \\
& =\int_{\Omega} u^{\prime \prime} \cdot u^{*} d x+\int_{\Omega} \sigma(u): \varepsilon\left(u^{*}\right) d x+\int_{\Gamma_{N}} m \cdot \nu u^{\prime} \cdot u^{*} d \Gamma-\int_{\gamma}[\sigma(u) \cdot \nu] \cdot u^{*} d \Gamma .
\end{aligned}
$$

In a similar manner, multiplying the second equation of (1) by $v^{*}$, and using integration by parts in $\omega$ and the boundary conditions, we obtain

$$
\begin{aligned}
0 & =\int_{\omega}\left[v^{\prime \prime}+\rho \partial^{4} v\right] v^{*} d s \\
& =\int_{\omega} v^{\prime \prime} v^{*} d s+\int_{\omega} \rho \partial^{2} v \partial^{2} v^{*} d s+\left[\rho \partial^{3} v v^{*}\right]_{0}^{l}+\left[\rho \partial^{2} v \partial v^{*}\right]_{0}^{l} \\
& =\int_{\omega} v^{\prime \prime} v^{*} d s+\int_{\omega} \rho \partial^{2} v \partial^{2} v^{*} d s-\rho \partial^{3} v(0) v^{*}(0) .
\end{aligned}
$$

Summing these two identities and taking into account the transmission condition on $\gamma$ we arrive at the identity

$$
(u, v)^{\prime \prime}+A(u, v)+B\left(u^{\prime}, v^{\prime}\right)=(0,0) \text { in } V^{\prime} .
$$

We now introduce the operators defined on $V \times V$ by

$$
\begin{aligned}
& \mathbb{A}\left((u, v),\left(u^{*}, v^{*}\right)\right)=\left(\left(-u^{*},-v^{*}\right), A(u, v)\right), \\
& \mathbb{B}\left((u, v),\left(u^{*}, v^{*}\right)\right)=\left((0,0), B\left(u^{*}, v^{*}\right)\right) .
\end{aligned}
$$

Setting

$$
X=\left((u, v),\left(u^{\prime}, v^{\prime}\right)\right)
$$

and

$$
\mathcal{A}=\mathbb{A}+\mathbb{B},
$$


the system (1) reduces to

$$
\left\{\begin{array}{l}
X^{\prime}+\mathcal{A} X=0 \\
X(0)=\left(\left(u_{0}, v_{0}\right),\left(u_{1}, v_{1}\right)\right)
\end{array}\right.
$$

Lemma 2.1. Under the above hypotheses, the operator $\mathcal{A}$ defined on $\mathcal{H} \times \mathcal{H}$ by (4), with domain

$$
\begin{gathered}
\mathcal{D}(\mathcal{A})=\left\{\left((u, v),\left(u^{*}, v^{*}\right)\right) \in V \times \mathcal{H}:\left(-\operatorname{div}(\sigma(u)), \partial^{4} v\right) \in \mathcal{H},\right. \\
\sigma(u) \cdot \nu+m \cdot \nu u^{*}=0 \quad \text { on } \Gamma_{N}, \\
\rho \partial^{3} v(0)+\alpha v^{*}(0)+\int_{\gamma}[\sigma(u) \nu] \cdot \theta d \Gamma=0, \\
\left.\partial v(0)=\partial^{2} v(l)=\partial^{3} v(l)=0\right\}
\end{gathered}
$$

is maximal dissipative. Moreover $D(\mathcal{A})$ is dense in $\mathcal{H} \times \mathcal{H}$.

The proof of this Lemma is quite standard (see for instance [12, section 2] or [17, Lemma 3.2]). The theory of linear semi-groups $[29,32]$ leads to Theorem 1.1. Note further that for initial data $\left(\left(u_{0}, v_{0}\right),\left(u_{1}, v_{1}\right)\right) \in D(\mathcal{A})$, the system $(1)$ has a unique strong solution $(u, v)$ satisfying

$$
(u, v) \in C^{2}([0, \infty) ; \mathcal{H}) \cap C^{1}([0, \infty) ; V) \cap C([0, \infty) ; D(\mathcal{A})) .
$$

\section{Proof of Theorem 1.2}

Deriving (3) in time and integrating by parts in space we readily see that

$$
E^{\prime}(t)=-\int_{\Gamma_{N}} m \cdot \nu\left|u^{\prime}(t)\right|^{2} d \Gamma-\alpha v^{\prime}(0, t)^{2}
$$

and consequently

$$
E(S)-E(T)=\int_{S}^{T}\left[\int_{\Gamma_{N}} m \cdot \nu\left|u^{\prime}(t)\right|^{2} d x+\alpha v^{\prime}(0, t)^{2}\right] d t,
$$

for all $0 \leq S \leq T<\infty$. This leads to the decay of the energy.

We will now obtain the exponential decay of this energy. For that purpose introduce the constant

$$
R_{0}=\max _{x \in \bar{\Omega}}\left(\sum_{k=1}^{n}\left(x_{k}-x_{0 k}\right)^{2}\right)^{1 / 2} .
$$

Let further $\mu$ be the smallest positive constant such that for all $u \in\left(H_{\Gamma_{D}}^{1}(\Omega)\right)^{n}$

$$
\int_{\Gamma_{N}}|u|^{2} d \Gamma \leq \mu^{2} \int_{\Omega} \sigma(u): \varepsilon(u) d x
$$

We start with two technical Lemmas: 
Lemma 3.1. Let $(u, v)$ be a strong solution of (1). Define

$$
M(u)=2(m \cdot \nabla) u+(n-1) u
$$

and

$$
N(v)=2(s-l) \partial v-v .
$$

Then we have

$$
\begin{aligned}
\|M(u)(t)\|_{\left(L^{2}(\Omega)\right)^{n}}^{2} \leq C E(t), & \forall t \geq 0, \\
\|N(v)(t)\|_{L^{2}(\omega)}^{2} \leq C E(t), & \forall t \geq 0,
\end{aligned}
$$

where, here and below, $C>0$ means a positive constant independent of $(u, v)$.

Proof. By integration by parts we have

$$
\begin{aligned}
\|M(u)\|_{\left(L^{2}(\Omega)\right)^{n}}^{2} & =\int_{\Omega}\left[|2(m \cdot \nabla) u|^{2}+(n-1)^{2}|u|^{2}+4(n-1) u \cdot(m \cdot \nabla) u\right] d x \\
& =\int_{\Omega}\left[|2(m \cdot \nabla) u|^{2}+(n-1)^{2}|u|^{2}+2(n-1) m \cdot \nabla\left(|u|^{2}\right)\right] d x \\
& =\int_{\Omega}\left[|2(m \cdot \nabla) u|^{2}+\left(1-n^{2}\right)|u|^{2}\right] d x+2(n-1) \int_{\Gamma} m \cdot \nu|u|^{2} d \Gamma \\
& \leq 4 R_{0}^{2} \int_{\Omega}|\nabla u|^{2} d x+2(n-1) \int_{\Gamma} m \cdot \nu|u|^{2} d \Gamma .
\end{aligned}
$$

We conclude using Korn's inequality since $\Gamma_{D}$ is not empty.

For the second estimate by integration by parts we have

$$
\|N(v)(t)\|_{L^{2}(\omega)}^{2} \leq 4 \int_{\omega}(s-l)^{2}(\partial v(s, t))^{2} d s+3 \int_{\omega} v^{2}(s, t) d s-2 l v^{2}(0, t) .
$$

But Poincaré's inequality leads to

$$
\int_{\omega}(\partial v(s, t))^{2} d s+\int_{\omega} v^{2}(s, t) d s \leq C\left(\int_{\omega}\left(\partial^{2} v(s, t)\right)^{2} d s+v^{2}(0, t)\right) .
$$

These two inequalities yield

$$
\|N(v)(t)\|_{L^{2}(\omega)}^{2} \leq C\left(E(t)+v^{2}(0, t)\right) .
$$

Now the assumption $\theta \neq 0$ and the transmission condition $u=\theta v$ on $\gamma$ lead to

$$
v^{2}(0, t) \leq \frac{1}{\int_{\gamma} \theta^{2} d \Gamma} \int_{\gamma}|u|^{2} d \Gamma,
$$

and by Korn's inequality we obtain

$$
v^{2}(0, t) \leq C \int_{\Omega} \sigma(u): \varepsilon(u) d \Gamma \leq C E(t) .
$$

This estimate in (6) leads to the conclusion. 
For $0 \leq T \leq \infty$, we set

$$
\begin{gathered}
Q=\Omega \times(0, T), \quad q=\omega \times(0, T) \\
\Sigma=\Gamma \times(0, T), \quad \Sigma_{D}=\Gamma_{D} \times(0, T), \quad \Sigma_{N}=\Gamma_{N} \times(0, T) .
\end{gathered}
$$

Lemma 3.2. If $\alpha \geq 0$, there exists a constant $C \geq 0$ such that for all $\varepsilon \in(0,1)$ and $T \geq 0$, we have

$$
\int_{0}^{T} \int_{\Gamma_{N}}|u|^{2} d \Gamma d t+\int_{0}^{T}|v(0, t)|^{2} d t \leq \frac{C}{\varepsilon} E(0)+\varepsilon \int_{0}^{T} E(t) d t .
$$

Proof. For $t \geq 0$, consider the solution $z=z(t)$ of (compare with [9, Lemma 5.2])

$$
\begin{cases}\operatorname{div}(\sigma(z))=0 & \text { in } \Omega \\ z=u & \text { on } \Gamma .\end{cases}
$$

This solution is characterized by $z=\omega+u$ where $\omega \in\left(H_{0}^{1}(\Omega)\right)^{n}$ is the unique solution of

$$
\int_{\Omega} \sigma(\omega): \varepsilon(v) d x=-\int_{\Omega} \sigma(u): \varepsilon(v) d x \quad \forall v \in\left(H_{0}^{1}(\Omega)\right)^{n} .
$$

This identity means that

$$
\int_{\Omega} \sigma(z): \varepsilon(v) d x=0 \quad \forall v \in\left(H_{0}^{1}(\Omega)\right)^{n} .
$$

Taking $v=z-u$ in this identity, we deduce that

$$
\int_{\Omega} \sigma(z): \varepsilon(u) d x=\int_{\Omega} \sigma(z): \varepsilon(z) d x \geq 0 .
$$

One easily shows that $z$ also satisfies (see [9, Lemma 5.2])

$$
\int_{\Omega} f \cdot z d x=-\int_{\Gamma} z \cdot\left(\sigma\left(v_{f}\right) \nu\right) d \Gamma, \quad \forall f \in\left(L^{2}(\Omega)\right)^{n},
$$

where $v_{f} \in\left(H_{0}^{1}(\Omega)\right)^{n}$ is the unique solution of

$$
\int_{\Omega} \sigma\left(v_{f}\right): \varepsilon(w) d x=\int_{\Omega} f \cdot w d x, \forall w \in\left(H_{0}^{1}(\Omega)\right)^{n} .
$$

Taking $f=z$ in the identity (9), we may write

$$
\int_{\Omega}|z|^{2} d x=-\int_{\Gamma} z \cdot\left(\sigma\left(v_{z}\right) \nu\right) d \Gamma .
$$


Since $z=u$ on $\Gamma_{N}, z=u=0$ on $\Gamma_{D}$, and $z=u=\theta v$ on $\gamma$, by Cauchy-Schwarz's inequality we obtain

$$
\int_{\Omega}|z|^{2} d x \leq C\left(\|u\|_{\left(L^{2}\left(\Gamma_{N}\right)\right)^{n}}+|v(0, t)|\right)\left\|\sigma\left(v_{z}\right) \nu\right\|_{\left(L^{2}(\Gamma)\right)^{n}}
$$

As the boundary $\Gamma$ is $C^{2}$, elliptic regularity results yield $v_{z} \in\left(H^{2}(\Omega)\right)^{n}$ with the estimate

$$
\left\|v_{z}\right\|_{\left(H^{2}(\Omega)\right)^{n}} \leq K\|z\|_{\left(L^{2}(\Omega)\right)^{n}},
$$

for some positive constant $K$. This estimate and a standard trace theorem lead to

$$
\left\|\sigma\left(v_{z}\right) \nu\right\|_{\left(L^{2}(\Gamma)\right)^{n}} \leq K_{1}\|z\|_{\left(L^{2}(\Omega)\right)^{n}}
$$

for some positive constant $K_{1}$. Inserting this estimate in (10) we arrive at

$$
\int_{\Omega}|z|^{2} d x \leq C\left(\int_{\Gamma_{N}}|u|^{2} d \Gamma+|v(0, t)|^{2}\right) .
$$

Since $z^{\prime}$ is solution of problem (7) with $u^{\prime}$ instead of $u$, the above arguments yield

$$
\int_{\Omega}\left|z^{\prime}\right|^{2} d x \leq C\left(\int_{\Gamma_{N}}\left|u^{\prime}\right|^{2} d \Gamma+\left|v^{\prime}(0, t)\right|^{2}\right) .
$$

In the same manner for $t \geq 0$, consider the solution $w=w(t)$ of

$$
\left\{\begin{array}{l}
\partial^{4} w=0 \quad \text { in } \omega \\
w(0)=v(0), \quad \partial w(0)=\partial v(0)=0, \quad \partial^{2} w(l)=\partial^{3} w(l)=0 .
\end{array}\right.
$$

This solution $w$ is characterized by $w=w_{1}+v$ where $w_{1} \in W$ is the unique solution of

$$
\int_{\omega} \partial^{2} w_{1} \partial^{2} k d s=-\int_{\omega} \partial^{2} v \partial^{2} k d s, \quad \forall k \in W,
$$

the Hilbert space $W$ (with the natural norm) being defined by

$$
W=\left\{k \in H^{2}(\omega): k(0)=\partial k(0)=\partial^{2} k(l)=\partial^{3} k(l)=0\right\} .
$$

As before this identity means that

$$
\int_{\omega} \partial^{2} w \partial^{2} k d s=0 \quad \forall k \in W
$$

and taking $k=w-v$ in this identity, we deduce that

$$
\int_{\omega} \partial^{2} v \partial^{2} w=\int_{\omega}\left(\partial^{2} w\right)^{2} d s \geq 0
$$


Let us also notice that $w$ satisfies

$$
\int_{\omega} g w d s=-w(0) \partial^{3} k_{g}(0), \quad \forall g \in L^{2}(\omega),
$$

where $k_{g} \in W$ is the unique solution of

$$
\int_{\omega} \partial^{2} k_{g} \partial^{2} k d s=\int_{\omega} g k d s, \forall k \in W .
$$

Taking $g=w$ in the identity (15), we may write

$$
\int_{\omega}|w|^{2} d s=-w(0) \partial^{3} k_{w}(0)
$$

and since $w(0)=v(0)$, we obtain

$$
\int_{\omega}|w|^{2} d s \leq|v(0, t)|\left|\partial^{3} k_{w}(0)\right|
$$

As $k_{w} \in H^{4}(\omega)$ with the estimate

$$
\left\|k_{w}\right\|_{H^{4}(\omega)} \leq K^{*}\|w\|_{L^{2}(\omega)},
$$

for some positive constant $K^{*}$, by the Sobolev embedding theorem we obtain

$$
\left|\partial^{3} k_{w}(0)\right| \leq K_{1}^{*}\|w\|_{L^{2}(\omega)}
$$

for some positive constant $K_{1}^{*}$. Inserting this estimate in (16) we arrive at

$$
\int_{\omega}|w|^{2} d s \leq C|v(0, t)|
$$

Since $w^{\prime}$ is solution of problem (13) with $v^{\prime}$ instead of $v$, the above arguments yield

$$
\int_{\omega}\left|w^{\prime}\right|^{2} d s \leq C\left|v^{\prime}(0, t)\right|
$$

Now using a standard trace theorem and Korn's inequality (

$$
\int_{\Gamma_{N} \cup \gamma}|z|^{2} d \Gamma \leq C \int_{\Omega} \sigma(z): \varepsilon(z) d x .
$$

Recalling that $z=u$ on $\Gamma_{N}$ and $z=u=\theta v$ on $\gamma$, we get

$$
\int_{\Gamma_{N}}|u|^{2} d \Gamma+|v(0, t)|^{2} \leq C \int_{\Omega} \sigma(z): \varepsilon(z) d x .
$$


This implies that

$$
\int_{\Gamma_{N}}|u|^{2} d \Gamma+|v(0, t)|^{2} \leq C\left(\int_{\Omega} \sigma(z): \varepsilon(z) d x+\rho \int_{\omega}\left(\partial^{2} w\right)^{2} d s\right) .
$$

Using the identities (8) and (14) we get

$$
\int_{\Gamma_{N}}|u|^{2} d \Gamma+|v(0, t)|^{2} \leq C\left(\int_{\Omega} \sigma(z): \varepsilon(u) d x+\rho \int_{\omega} \partial^{2} v \partial^{2} w\right) .
$$

Integrating this identity for $t \in(0, T)$, we find

$$
\int_{\Sigma_{N}}|u|^{2} d \Gamma d t+\int_{0}^{T}|v(0, t)|^{2} d t \leq C\left(\int_{Q} \sigma(u): \varepsilon(z) d x d t+\rho \int_{q} \partial^{2} v \partial^{2} w d s d t\right) .
$$

By integration by parts, we get

$$
\begin{aligned}
\int_{\Sigma_{N}}|u|^{2} d \Gamma d t+\int_{0}^{T}|v(0, t)|^{2} d t \leq & C\left(-\int_{Q} \operatorname{div} \sigma(u) \cdot z d x d t+\rho \int_{q} \partial^{4} v w d s d t\right. \\
& \left.+\int_{\Sigma} \sigma(u) \nu \cdot z d \Gamma d t+\rho \int_{0}^{T} w(0, t) \partial^{3} v(0, t) d t\right) .
\end{aligned}
$$

As $z=u$ on $\Sigma_{N}, z=0$ on $\Sigma_{D}, z=\theta v(0, t)$ on $\gamma \times(0, T)$, and $w(0)=v(0, t)$, using the boundary conditions on $\Sigma_{N}$ and on $\gamma \times(0, T)$ for $u$ we may write

$$
\int_{\Sigma} \sigma(u) \nu \cdot z d \Gamma d t=\int_{\Sigma_{N}} m \cdot \nu u^{\prime} u d \Gamma d t-\int_{0}^{T} v(0, t)\left(\rho \partial^{3} v(0, t)+\alpha v^{\prime}(0, t)\right) d t .
$$

Inserting this identity in the last one and using the first and second identities of (1), we arrive at

$$
\begin{aligned}
\int_{\Sigma_{N}}|u|^{2} d \Gamma+\int_{0}^{T}|v(0, t)|^{2} d t \leq C & \left(-\int_{Q} u^{\prime \prime} \cdot z d x d t-\int_{q} v^{\prime \prime} w d s d t\right. \\
& \left.+\int_{\Sigma_{N}} m \cdot \nu u^{\prime} u d \Gamma d t-\alpha \int_{0}^{T} v(0, t) v^{\prime}(0, t) d t\right) .
\end{aligned}
$$

Now integrating by parts in time, we obtain

$$
\begin{aligned}
\int_{\Sigma_{N}}|u|^{2} d \Gamma+\int_{0}^{T}|v(0, t)|^{2} d t \leq C\left(\int_{Q} u^{\prime} \cdot z^{\prime} d x d t+\int_{q} v^{\prime} w^{\prime} d s d t\right. \\
\left.\quad-\left.\int_{\Omega} z u^{\prime}\right|_{0} ^{T}-\left.\int_{\omega} w v^{\prime}\right|_{0} ^{T}+\int_{\Sigma_{N}} m \cdot \nu u^{\prime} u d \Gamma d t-\alpha \int_{0}^{T} v(0, t) v^{\prime}(0, t) d t\right) .
\end{aligned}
$$


Fix an arbitrary $\varepsilon_{0} \geq 0$. Using several times (5), (11), (12), (17), (18), and Young's inequality, we can estimate the different integrals of the right-hand side of the above inequality as follows:

$$
\begin{aligned}
& \int_{\Sigma_{N}} m \cdot \nu u u^{\prime} d \Sigma \leq \varepsilon_{0} \int_{\Sigma_{N}}|u|^{2} d \Sigma+\frac{1}{4 \varepsilon_{0}} \int_{\Sigma_{N}} m \cdot \nu\left|u^{\prime}\right|^{2} d \Sigma \\
& \leq 2 \varepsilon_{0} \mu^{2} \int_{0}^{T} E(t) d t+\frac{1}{4 \varepsilon_{0}} E(0), \\
& \int_{Q} z^{\prime} u^{\prime} d x d t \leq \varepsilon_{0} \int_{Q}\left|u^{\prime}\right|^{2} d x d t+\frac{1}{4 \varepsilon_{0}} \int_{Q}\left|z^{\prime}\right|^{2} d x d t \\
& \leq 2 \varepsilon_{0} \int_{0}^{T} E(t) d t+\frac{C}{4 \varepsilon_{0}} E(0), \\
& \int_{q} w^{\prime} v^{\prime} d x d t \leq 2 \varepsilon_{0} \int_{0}^{T} E(t) d t+\frac{C}{4 \varepsilon_{0}} E(0) \\
& -\left.\int_{\Omega} z u^{\prime}\right|_{0} ^{T} \leq 4\left(1+C \mu^{2}\right) E(0), \\
& -\left.\int_{\omega} w v^{\prime}\right|_{0} ^{T} \leq C E(0) \\
& -\alpha \int_{0}^{T} v(0, t) v^{\prime}(0, t) d t \leq \frac{1}{\varepsilon_{0}} \alpha \int_{0}^{T}\left|v^{\prime}(0, t)\right|^{2} d t+\varepsilon_{0} \int_{0}^{T}|v(0, t)|^{2} d t \\
& \leq \frac{1}{\varepsilon_{0}} E(0)+\varepsilon_{0} \int_{0}^{T}|v(0, t)|^{2} d t .
\end{aligned}
$$

Using these different estimates in (19), we arrive at the requested estimate by choosing $\varepsilon_{0}$ appropriately.

Proof of Theorem 1.2. Without loss of generality we can assume that

$$
\left(\frac{l}{2}+\int_{\gamma} m \cdot \nu|\theta(x)| d \Gamma\right) \leq 0
$$

Indeed if (20) is not satisfied, we can use the following scaling argument: For a parameter $\beta>0$ fixed later on, let us set

$$
\hat{v}(\hat{s}, t)=v(\beta \hat{s}, t) \text { on } \hat{\omega},
$$

where

$$
\hat{\omega}=\{a+\hat{s} \nu(a): 0<\hat{s}<\hat{l}\}
$$


$\hat{l}=l / \beta$ being the length of $\hat{\omega}$. We then see that the pair $(u, \hat{v})$ is solution of $(1)$ with $\omega\left(\right.$ resp. $\rho$ ) replaced by $\hat{\omega}$ (resp. $\hat{\rho}=\beta^{-4} \rho$ ). For this new system, the condition (20) is equivalent to

$$
\left(\frac{l}{2 \beta}+\int_{\gamma} m \cdot \nu|\theta(x)| d \Gamma\right) \leq 0,
$$

which holds if $\beta$ is chosen sufficiently large, namely if

$$
\beta \geq-\frac{l}{2 \int_{\gamma} m \cdot \nu|\theta(x)| d \Gamma}
$$

For a fixed $\beta$, we further notice that

$$
\min \{1, \beta\} \hat{E}(t) \leq E(t) \leq \max \{1, \beta\} \hat{E}(t),
$$

where $\hat{E}(t)$ is the energy of the new system:

$$
\hat{E}(t)=\frac{1}{2} \int_{\Omega}\left\{\left|u^{\prime}\right|^{2}+\sigma(u): \varepsilon(u)\right\} d x+\frac{1}{2} \int_{\hat{\omega}}\left\{\left|\hat{v}^{\prime}\right|^{2}+\hat{\rho}\left|\hat{\partial}^{2} \hat{v}\right|^{2}\right\} d \hat{s} .
$$

Consequently the exponential stability of the energy $E$ is equivalent to the exponential stability of the energy $\hat{E}$. Therefore if (20) does not hold, it suffices to consider the new system for $(u, \hat{v})$ for a fixed $\beta$ satisfying (21) and the exponential stability of this new system (proved below) will imply the exponential stability of the original system

Assume first that $(u, v)$ is a strong solution of (1).

If $\bar{\Gamma}_{D} \cap \bar{\Gamma}_{N}=\emptyset$, then multiplying the first identity of (1) by

$$
M(u)=2(m \cdot \nabla) u+(n-1) u
$$

and integrating by parts on $Q$ we obtain

$$
\begin{aligned}
0=\left.\left(u^{\prime}, M(u)\right)\right|_{0} ^{T} & +\int_{Q}\left|u^{\prime}\right|^{2} d x d t-\int_{\Sigma_{N}} m \cdot \nu\left|u^{\prime}\right|^{2} d \Sigma \\
& -\int_{\gamma \times[0, T]} m \cdot \nu\left|u^{\prime}\right|^{2} d s(x) d t+\int_{Q} \sigma(u): \varepsilon(u) d x d t \\
& -\int_{\Sigma}[(\sigma(u) \nu) \cdot M(u)-(m \cdot \nu) \sigma(u): \varepsilon(u)] d \Sigma .
\end{aligned}
$$

If $\Gamma_{D} \cap \Gamma_{N} \neq \emptyset$, then applying [3, Theorem 4.1], we have

$$
\begin{aligned}
0 \geq\left.\left(u^{\prime}, M(u)\right)\right|_{0} ^{T} & +\int_{Q}\left|u^{\prime}\right|^{2} d x d t-\int_{\Sigma_{N}} m \cdot \nu\left|u^{\prime}\right|^{2} d \Sigma \\
- & \int_{\gamma \times[0, T]} m \cdot \nu\left|u^{\prime}\right|^{2} d s(x) d t+\int_{Q} \sigma(u): \varepsilon(u) d x d t \\
& -\int_{\Sigma}[(\sigma(u) \nu) \cdot M(u)-(m \cdot \nu) \sigma(u): \varepsilon(u)] d \Sigma .
\end{aligned}
$$


Similarly multiplying the second identity of (1) by $N(v)$ and integrating by parts on $q$ we obtain

$$
\begin{aligned}
0=2 \int_{0}^{T} & \int_{\omega}\left|v^{\prime}\right|^{2}-l \int_{0}^{T}\left|v^{\prime}(0, t)\right|^{2} d t \\
& +\left.\int_{\omega} v^{\prime} N(v)\right|_{0} ^{T}+2 \rho \int_{0}^{T} \int_{0}^{l}\left(\partial^{2} v\right)^{2} d t+2 l \rho \int_{0}^{T} \partial^{3} v(0, t) \partial v(0, t) \\
& +\rho \int_{0}^{T} \partial^{3} v(0, t) v(0, t) d t
\end{aligned}
$$

These two identities (or inequalities if $\bar{\Gamma}_{D} \cap \bar{\Gamma}_{N} \neq \emptyset$ ) allow to obtain

$$
\int_{0}^{T} E(t) d t \leq \int_{\Sigma_{N}} m \cdot \nu\left|u^{\prime}\right|^{2}+\sum_{i=1}^{4} I_{i}
$$

where

$$
\begin{aligned}
I_{1} & =-\left.\int_{\Omega}\left(u^{\prime}, M(u)\right)\right|_{0} ^{T}-\left.\frac{1}{2} \int_{\omega} N(v) v^{\prime}\right|_{0} ^{T}, \\
I_{2} & =\int_{\Sigma_{N} \cup \Sigma_{D}}[(\sigma(u) \nu) \cdot M(u)-(m \cdot \nu) \sigma(u): \varepsilon(u)] d \Sigma, \\
I_{3} & =\int_{\gamma \times(0, T)} m \cdot \nu\left|u^{\prime}\right|^{2} d \Gamma d t+\frac{l}{2} \int_{0}^{T} v^{\prime 2}(0, t) d t, \\
I_{4} & =\int_{\gamma \times(0, T)}[(\sigma(u) \nu) \cdot M(u)-(m \cdot \nu) \sigma(u): \varepsilon(u)] d \Sigma-\frac{1}{2} \rho \int_{0}^{T} \partial^{3} v(0, t) v(0, t) d t .
\end{aligned}
$$

Lemma 3.1 yields

$$
I_{1} \leq C E(0)
$$

As in $[1,9]$ using local coordinates systems we obtain the estimate

$$
I_{2} \leq C\left(E(0)+\int_{\Sigma_{N}}\left(|u|^{2}+\left|u^{\prime}\right|^{2}\right) d \Sigma\right)
$$

Using the boundary condition $u=\theta v$ on $\gamma \times(0, T)$ in system (1) and the condition (20), we get

$$
I_{3}=\left(\frac{l}{2}+\int_{\gamma} m \cdot \nu|\theta(x)|^{2}\right) \int_{0}^{T} v^{\prime 2}(0, t) d t \leq 0
$$


Again using the boundary condition on $\gamma \times(0, T)$ in system (1) we may write

$$
\begin{aligned}
I_{4}=\int_{\gamma \times(0, T)}[(\sigma(u) \nu) \cdot M(u)-(m \cdot \nu) \sigma(u): \varepsilon(u)] d \Sigma & \\
& +\frac{1}{2} \int_{\gamma \times(0, T)}(\sigma(u) \nu) \cdot \theta v(0, t) d \Sigma+\frac{\alpha}{2} \int_{0}^{T} v^{\prime}(0, t) v(0, t) d t .
\end{aligned}
$$

The estimation of $I_{4}$ is also based on the use of local coordinates systems (cf. [1]). Namely for all $x \in \Gamma$, we denote by $\pi(x)$ the orthogonal projection on the tangent hyperplane $T_{x}(\Gamma)$. Any vector field $v: \bar{\Omega} \rightarrow \mathbb{R}^{n}$ will be split up as follows:

$$
v(x)=v_{T}(x)+v_{\nu}(x) \nu(x),
$$

where $v_{T}(x)=\pi(x) v(x)$ is the tangential component of $v$ and $v_{\nu}(x)=v(x) \cdot \nu(x)$. We further denote by $\partial_{\nu} v=\nu \cdot \nabla v$, the normal derivative of $v$ and by $\nabla_{T} v=\nabla v-\partial_{n} v$ the tangential gradient of $v$. For further uses, we set $\partial_{T} v=\bar{\nabla}_{T} v$, the tangential derivation of $v$, where $\bar{\tau}$ means the transposed matrix of the matrix $\tau$. Similarly for a vector $v, \bar{v}$ will mean its transposed vector.

Following [15] or [33], the strain tensor is written as follows:

$$
\varepsilon(v)=\varepsilon_{T}(v)+\nu \overline{\varepsilon_{S}(v)}+\varepsilon_{S}(v) \bar{\nu}+\varepsilon_{\nu}(v) \nu \bar{\nu} \quad \text { on } \Gamma,
$$

with

$$
\begin{aligned}
2 \varepsilon_{T}(v) & =\pi\left(\partial_{T} v_{T}\right) \pi+\pi \overline{\partial_{T} v_{T}} \pi+2 v_{\nu} \partial_{T} \nu, \\
2 \varepsilon_{S}(v) & =\partial_{\nu} v_{T}+\nabla_{T} v_{\nu}-\left(\partial_{T} \nu\right) v_{T}, \\
\varepsilon_{\nu}(v) & =\partial_{\nu} v_{\nu},
\end{aligned}
$$

where $\left(\partial_{T} \nu\right)$ is the curvature operator of $\Gamma$. Similarly the stress tensor may be written

$$
\sigma(v)=\sigma_{T}(v)+\nu \overline{\sigma_{S}(v)}+\sigma_{S}(v) \bar{\nu}+\sigma_{\nu}(v) \nu \bar{\nu} \quad \text { on } \Gamma,
$$

where $\sigma_{T}(v)$ is an endomorphism on the tangent hyperplane, $\sigma_{S}(v)$ is a tangent vector field and $\sigma_{\nu}(v)$ is a scalar field.

These splittings allow to write

$$
\begin{aligned}
& \varepsilon(v): \varepsilon(v)=\varepsilon_{T}(v): \varepsilon_{T}(v)+2\left|\varepsilon_{S}(v)\right|^{2}+\left|\varepsilon_{\nu}(v)\right|^{2} \quad \text { on } \Gamma, \\
& \sigma(v): \varepsilon(v)=\sigma_{T}(v): \varepsilon_{T}(v)+2 \overline{\sigma_{S}(v)} \varepsilon_{S}(v)+\sigma_{\nu}(v) \varepsilon_{\nu}(v) \quad \text { on } \Gamma \text {. }
\end{aligned}
$$

Using these local coordinates systems and the boundary condition on $\gamma \times(0, T)$ in system (1) we obtain

$$
\begin{aligned}
\sigma(u) \nu & =\sigma_{S}(u)+\sigma_{\nu}(u) \nu & & \text { on } \gamma \times(0, T), \\
M(u) & =2(m \cdot \nu) \partial_{\nu} u+v_{1}(\theta) v(0, t) & & \text { on } \gamma \times(0, T),
\end{aligned}
$$


for some vector valued function $v_{1}(\theta)$ (depending on $\theta$ and its tangential gradient). This yields

$$
\begin{aligned}
\sigma(u) \nu \cdot M(u)= & \overline{\sigma(u) \nu} M(u) \\
= & 2(m \cdot \nu)\left(\bar{\sigma}_{S}(u)+\sigma_{\nu}(u) \bar{\nu}\right) \partial_{\nu} u+\sigma(u) \nu \cdot v_{1}(\theta) v(0, t) \\
= & 2(m \cdot \nu)\left(\bar{\sigma}_{S}(u) \partial_{\nu} u_{T}+\sigma_{\nu}(u) \bar{\nu} \partial_{\nu} u_{\nu}\right) \\
& +\sigma(u): C_{1}(\theta) v(0, t) \text { on } \gamma \times(0, T),
\end{aligned}
$$

for some matrix valued function $C_{1}(\theta)$ (depending on $\theta$ and its tangential gradient).

On the other hand, we recall that

$$
\sigma(u): \varepsilon(u)=\sigma_{T}(u): \varepsilon_{T}(u)+2 \overline{\sigma_{S}(u)} \varepsilon_{S}(u)+\sigma_{\nu}(u) \varepsilon_{\nu}(u) \quad \text { on } \gamma \times(0, T),
$$

and again using the boundary condition, we obtain

$$
\sigma(u): \varepsilon(u)=\left(\bar{\sigma}_{S}(u) \partial_{\nu} u_{T}+\sigma_{\nu}(u) \bar{\nu} \partial_{\nu} u_{\nu}\right)+\sigma(u): C_{2}(\theta) v(0, t) \quad \text { on } \gamma \times(0, T) .
$$

All together we arrive at

$$
\begin{aligned}
(\sigma(u) \nu) \cdot M(u)-(m \cdot \nu) \sigma(u): \varepsilon(u)=(m \cdot \nu) \sigma(u) & : \varepsilon(u) \\
& +\sigma(u): C_{3}(\theta) v(0, t) \quad \text { on } \gamma \times(0, T) .
\end{aligned}
$$

Inserting this identity into (22), we obtain

$$
\begin{aligned}
I_{4}= & \int_{\gamma \times(0, T)}(m \cdot \nu) \sigma(u): \varepsilon(u) d \Sigma \\
& \quad+\int_{\gamma \times(0, T)}\left[\sigma(u): C_{3}(\theta)+\frac{1}{2}(\sigma(u) \nu) \cdot \theta\right] v(0, t) d \Sigma+\frac{\alpha}{2} \int_{0}^{T} v^{\prime}(0, t) v(0, t) d t .
\end{aligned}
$$

By Young's inequality we obtain

$$
\begin{aligned}
I_{4} \leq \int_{\gamma \times(0, T)}(m \cdot \nu) \sigma(u): \varepsilon(u) d \Sigma d t & \\
& +\epsilon \int_{\gamma \times(0, T)}|\sigma(u)|^{2} d \Sigma d t+\frac{C}{\epsilon} \int_{0}^{T}|v(0, t)|^{2} d t \\
& \quad+\alpha \int_{0}^{T}\left|v^{\prime}(0, t)\right|^{2} d t, \forall \epsilon \in(0,1) .
\end{aligned}
$$

Now using the assumption (2), we may write

$$
|\sigma(u)|^{2} \leq C|\varepsilon(u)|^{2} \leq C \sigma(u): \varepsilon(u) .
$$


Therefore reminding that $m \cdot \nu<-\alpha_{0}<0$ on $\gamma$, by fixing $\epsilon<\frac{C \alpha_{0}}{2}$, we obtain

$$
I_{4} \leq \int_{\gamma \times(0, T)} \frac{m \cdot \nu}{2} \sigma(u): \varepsilon(u) d \Sigma d t+C \int_{0}^{T}|v(0, t)|^{2} d t+\alpha \int_{0}^{T}\left|v^{\prime}(0, t)\right|^{2} d t .
$$

Since $m \cdot \nu \leq 0$ on $\gamma$, we conclude that

$$
I_{4} \leq C \int_{0}^{T}|v(0, t)|^{2} d t+\alpha \int_{0}^{T}\left|v^{\prime}(0, t)\right|^{2} d t
$$

The estimates on $I_{i}, i=1, \ldots, 4$ yield

$$
\begin{aligned}
2 \int_{0}^{T} E(t) d t \leq C\left(E(0)+\int_{\Sigma_{N}} m \cdot \nu\left|u^{\prime}\right|^{2} d \Sigma\right. & \left.+\alpha \int_{0}^{T}\left|v^{\prime}(0, t)\right|^{2} d t\right) \\
& +C \int_{\Sigma_{N}}|u|^{2} d \Sigma+C(\theta) \int_{0}^{T} v^{2}(0, t) d t .
\end{aligned}
$$

By Lemma 3.2 the above estimate (23) becomes

$$
\begin{aligned}
2 \int_{0}^{T} E(t) d t \leq C\left(E(0)+\int_{\Sigma_{N}} m \cdot \nu\left|u^{\prime}\right|^{2} d \Sigma+\alpha \int_{0}^{T}\left|v^{\prime}(0, t)\right|^{2} d t\right) & \\
& +\frac{C}{\varepsilon} E(0)+\varepsilon \int_{0}^{T} E(t) d t
\end{aligned}
$$

for any $\varepsilon>0$. By choosing $\varepsilon$ small enough, we arrive at the observability estimate

$$
\int_{0}^{T} E(t) d t \leq C\left(E(0)+\int_{\Sigma_{N}} m \cdot \nu\left|u^{\prime}\right|^{2} d \Sigma+\alpha \int_{0}^{T}\left|v^{\prime}(0, t)\right|^{2} d t\right) .
$$

This estimate remains valid for weak solutions by a density argument. The conclusion now follows from this estimate as shown in [26, Theorem 3.3].

Acknowledgements. We thank the referee of our paper whose remarks allowed us to improve the statement and the proof of Theorem 1.2.

\section{References}

[1] R. Bey, A. Heminna, and J.-P. Lohéac, Boundary stabilization of a linear elastodynamic system with variable coefficients, Electron. J. Differential Equations 78 (2001), 1-23.

[2] R. Brossard and J.-P. Lohéac, Stabilisation frontière du système élastodynamique dans un polygone plan, C. R. Math. Acad. Sci. Paris 338 (2004), no. 3, 213-218 (French, with English and French summaries). 
[3] _ Boundary stabilization of elastodynamic systems Part I: Rellich-type relations for a mixed boundary problem in elasticity, Univ. de Lyon, 2004, preprint MAPLY.

[4] B. Dehman and L. Robbiano, La propriété du prolongement unique pour un système elliptique. Le système de Lamé, J. Math. Pures Appl. (9) 72 (1993), no. 5, 475-492.

[5] M. Eller, J. E. Lagnese, and S. Nicaise, Decay rates for solutions of a Maxwell system with nonlinear boundary damping, Comput. Appl. Math. 21 (2002), no. 1, 135-165.

[6] A. Guesmia, Existence globale et stabilisation frontière non linéaire d'un système d'élasticité, Portugal. Math. 56 (1999), no. 3, 361-379 (French, with English and French summaries).

[7] S. W. Hansen, Exponential energy decay in a linear thermoelastic rod, J. Math. Anal. Appl. 167 (1992), no. 2, 429-442.

[8] A. Heminna, S. Nicaise, and A. Sène, Stabilisation d'un système de la thermoélasticité anisotrope avec feedbacks non linéaires, C. R. Math. Acad. Sci. Paris 339 (2004), no. 8, 561-566 (French, with English and French summaries).

[9] Stabilization of a system of anisotropic thermoelasticity by nonlinear boundary and internal feedbacks, Quart. Appl. Math. 63 (2005), no. 3, 429-453.

[10] M. A. Horn, Implications of sharp trace regularity results on boundary stabilization of the system of linear elasticity, J. Math. Anal. Appl. 223 (1998), no. 1, 126-150.

[11] V. Komornik, Exact controllability and stabilization - The multiplier method, Research in Applied Mathematics, Masson, Paris, 1994

[12] J. Lagnese, Boundary stabilization of linear elastodynamic systems, SIAM J. Control Optim. 21 (1983), no. 6, 968-984.

[13] J. E. Lagnese, G. Leugering, and E. J. P. G. Schmidt, Modeling, analysis and control of dynamic elastic multi-link structures, Systems \& Control: Foundations \& Applications, Birkhäuser Boston Inc., Boston, MA, 1994.

[14] G. Lebeau and E. Zuazua, Decay rates for the three-dimensional linear system of thermoelasticity, Arch. Ration. Mech. Anal. 148 (1999), no. 3, 179-231.

[15] K. Lemrabet, Étude de divers problèmes aux limites de Ventcel d'origine physique ou mécanique in des domaines non réguliers, Ph.D. thesis, Université des Sciences et de Technologie Haouari Boumediene, Alger, 1987.

[16] W.-J. Liu, Partial exact controllability and exponential stability in higher-dimensional linear thermoelasticity, ESAIM Control Optim. Calc. Var. 3 (1998), 23-48.

[17] W.-J. Liu and E. Zuazua, Uniform stabilization of the higher-dimensional system of thermoelasticity with a nonlinear boundary feedback, Quart. Appl. Math. 59 (2001), no. 2, 269-314.

[18] Z. Liu and S. M. Zheng, Exponential stability of the semigroup associated with a thermoelastic system, Quart. Appl. Math. 51 (1993), no. 3, 535-545.

[19] J.-P. Lohéac, Problèmes elliptiques à données peu régulières applications, thèse d'habilitation, Ecole Centrale Lyon, 2002.

[20] J. E. Muñoz Rivera, Decomposition of the displacement vector field and decay rates in linear thermoelasticity, SIAM J. Math. Anal. 24 (1993), no. 2, 390-406.

[21] $\ldots$ Asymptotic behaviour in n-dimensional thermoelasticity, Appl. Math. Lett. 10 (1997), no. $5,47-53$.

[22] G. Nakamura and J.-N. Wang, The limiting absorption principle for the two-dimensional inhomogeneous anisotropic elasticity system, Trans. Amer. Math. Soc., to appear.

[23] _ Unique continuation for the two-dimensional anisotropic elasticity system and its applications to inverse problems, Trans. Amer. Math. Soc., to appear. 
[24] K. Narukawa, Boundary value control of thermoelastic systems, Hiroshima Math. J. 13 (1983), no. $2,227-272$.

[25] S. Nicaise, Stability and controllability of the electromagneto-elastic system, Port. Math. (N.S.) 60 (2003), no. 1, 37-70.

[26] Stability and controllability of an abstract evolution equation of hyperbolic type and concrete applications, Rend. Mat. Appl. (7) 23 (2003), no. 1, 83-116.

[27] S. Nicaise and A. Sène, Stabilisation non linéaire d'un système de la thermoélasticité isotropes (2003), preprint MACS 03-12.

[28] L. A. F. de Oliveira, Exponential decay in thermoelasticity, Commun. Appl. Anal. 1 (1997), no. $1,113-118$.

[29] A. Pazy, Semigroups of linear operators and applications to partial differential equations, Applied Mathematical Sciences, vol. 44, Springer-Verlag, New York, 1983.

[30] J.-P. Puel and E. Zuazua, Exact controllability for some models of multidimensional vibrating structures, Mathematics, climate and environment (Madrid, 1991), RMA Res. Notes Appl. Math., vol. 27, Masson, Paris, 1993, pp. 288-295.

[31] _ Exact controllability for a model of a multidimensional flexible structure, Proc. Roy. Soc. Edinburgh Sect. A 123 (1993), no. 2, 323-344.

[32] R. E. Showalter, Monotone operators in Banach space and nonlinear partial differential equations, Mathematical Surveys and Monographs, vol. 49, American Mathematical Society, Providence, RI, 1997.

[33] R. Valid, La mécanique des milieux continus et le calcul des structures, Eyrolles, Paris, 1977. 